\title{
La inmigración y el género como factores clave de la movilidad educativa en España
}

\author{
Rafael Granell \\ Amadeo Fuenmayor \\ Universidad de Valencia. Departamento de Economía Aplicada
}

Mauro Mediavilla

Universidad de Valencia. Departamento de Economía Aplicada / Institut d'Economia de Barcelona (IEB)

rafael.granell@uv.es; amadeo.fuenmayor@uv.es; mauro.mediavilla@uv.es

Recepción: 03-04-2016

Aceptación: 09-09-2016

\section{Resum}

En este trabajo analizamos los determinantes del logro educativo para la población inmigrante en España, así como su grado de movilidad intergeneracional educativa, comparando estos resultados con el resto de residentes en nuestro país. Como fuente de información, utilizamos la encuesta EU-SILC de 2011, que incluye un módulo de transmisión intergeneracional de pobreza. Gracias a este módulo, podemos conocer el nivel académico de los progenitores y compararlo con el alcanzado por sus hijos. Para analizar los determinantes del logro educativo, utilizamos una estimación de máxima verosimilitud, teniendo en cuenta variables del propio individuo, de su familia y de su entorno, mientras que el estudio de movilidad educativa está basado en las tres técnicas más utilizadas en este tipo de trabajos: correlación, regresión y matrices de transición. Respecto al logro educativo, la estimación efectuada indica que la condición de inmigrante tiene un efecto negativo sobre el nivel académico alcanzado. Además, el nivel académico de los inmigrantes está muy relacionado con el de sus madres y con la situación económica del hogar donde crecieron. En cuanto a la movilidad intergeneracional, se observa una clara mejoría en la situación de todos los individuos analizados respecto a sus madres. Sin embargo, el grado de movilidad es muy diferente en función del género. Los hombres presentan unos indicadores similares independientemente de su lugar de nacimiento, mientras que las mujeres inmigrantes tienen una movilidad inferior a las de origen español.

Palabras clave: movilidad educativa; inmigración; logro educativo; diferencias de género 
Abstract. Immigration and gender as key factors of educational mobility in Spain

This paper analyzes the determinants of educational attainment among immigrants in Spain and their degree of intergenerational educational mobility compared to Spanish individuals. The EU-SILC survey of 2011, which includes a module on intergenerational transmission of poverty, is used as a source of information. This module allows identifying the educational attainment of parents and comparing it with the educational attainment of their children. To analyze the determinants of educational attainment, a maximum likelihood estimation is used taking into account variables related to the individual, his/ her family and his/her environment. The study of intergenerational mobility is based on three widely used techniques in the literature: correlation, regression and transition matrices. With respect to educational attainment, the estimation indicates that immigrant status has a negative effect on educational attainment. In addition, the educational level of immigrants is closely related to the educational level of their mothers and the economic situation of the home where they grew up. Educational mobility is high in all the groups observed, but is very different according to gender. Men have similar indicators regardless of their place of birth, while immigrant women have less mobility than Spanish women.

Keywords: educational mobility; immigration; educational attainment; gender differences

\section{Sumari}

1. Introducción 4. Movilidad intergeneracional educativa 2. Datos de los inmigrantes

3. Determinantes del nivel educativo de 5. Conclusiones

los inmigrantes Referencias bibliográficas

\section{Introducción}

El fenómeno de la inmigración responde a motivaciones muy variadas: inmigración económica, reunificación familiar, inmigración humanitaria (por asilo o refugio), inmigración estudiantil y adquisición (y pérdida) de la ciudadanía (Beine et al., 2014), y sus circunstancias son muy cambiantes. España es un buen ejemplo de ello, ya que, en las últimas décadas, el fenómeno migratorio ha sufrido fuertes cambios. Según Eurostat, en 2012, el flujo neto de inmigración a nuestro país superó las 304.000 personas, cifra muy por encima de lo que podría esperarse tan solo unos años antes. Este cambio histórico se ha traducido en un nivel muy importante de población inmigrante, un 13,2\%, claramente por encima del $9 \%$ de media de la UE (Eurostat, 2015). A diferencia de otros estados europeos, el caso español destaca por el elevado peso de la inmigración procedente de países de fuera de la UE, tanto en flujos (en 2012 un $62,1 \%$ proceden de fuera de la UE, frente a un $31,1 \%$ procedente de países de la UE) como en cantidad de población inmigrante. Tampoco es desdeñable el retorno de individuos nacidos en España (un 6,8\% del total de inmigrantes en 2012). Por otro lado, España destaca en el entorno europeo 
por la escasa antigüedad del fenómeno, al ser un territorio que se convierte en receptor neto de inmigración muy tardíamente.

En las últimas décadas, el fenómeno de la inmigración se ha intensificado considerablemente en España (Arango, 2004; Garrido, 2005; Requena y Reher, 2009). En cambio, históricamente siempre había sido un país con una fuerte tradición de emigración. Sin embargo, a partir de mediados de la década de 1980, básicamente se ha convertido en receptor de población extranjera. Desde finales de la década de 1990, ha recibido una tasa superior de inmigrantes a la de cualquier país europeo (Carrasco et al., 2008). Entre 1998 y 2009, la participación de los ciudadanos nacidos fuera de España pasó de un $4 \%$ a un $17 \%$, con un flujo de 4,5 millones de inmigrantes (González y Ortega, 2011). Estos flujos migratorios se originaron fundamentalmente a causa de un sólido crecimiento económico, de factores de afinidad cultural (es el caso de una gran parte de la población procedente de Latinoamérica) y también de acuerdos especiales que permitían a los ciudadanos de las antiguas colonias entrar en el país sin visado (Ecuador supone un 5,3\% de personas foráneas, y Colombia, un 4,4\%). Por otro lado, la afinidad lingüística ha sido un claro factor de atracción para los rumanos (De la Rica et al., 2014). Todo esto explica el elevado porcentaje de inmigrantes entre la población, así como el bajo peso de los que son de segunda generación. En 2013, los países con mayor presencia son Rumanía (un 15,2\%) y Marruecos (un 15\%) (Eurostat, 2015). Asimismo, una parte importante de la inmigración procede de países del norte de Europa (en 2013, el colectivo de inmigrantes originarios del Reino Unido representaba a un 6,2\% del total, aunque también eran importantes las colonias de personas procedentes de los países nórdicos) y está muy relacionada con la población jubilada.

El nivel académico alcanzado por los integrantes de este colectivo puede ser diferente al del resto de ciudadanos, principalmente por dos motivos. En primer lugar, los inmigrantes de primera generación provienen de países con un sistema educativo distinto al español y su nivel formativo, para la misma edad, no tiene por qué coincidir con el de las personas nacidas en España. En segundo lugar, el nivel educativo de los inmigrantes de segunda generación también puede verse influido por las características sociales, económicas y culturales de sus progenitores. Por lo tanto, conocer la existencia de condicionantes específicos para este colectivo tiene una amplia relevancia, ya que permitiría diseñar mejores políticas docentes de cara a lograr un sistema pedagógico más intercultural e inclusivo que considerara el pluralismo un elemento enriquecedor de la cultura propia.

El primer objetivo de este trabajo consiste en estudiar los determinantes del nivel educativo de las personas residentes en España y analizar si hay diferencias sustanciales entre la población inmigrante y el resto de ciudadanos. En el estudio de estos determinantes, nos centraremos en la influencia que tiene el nivel educativo de los padres sobre el de los hijos, como paso previo al segundo objetivo de este trabajo: el análisis de la movilidad intergeneracional académica. 
Los estudios de movilidad intergeneracional son muy habituales en la literatura y abarcan diferentes disciplinas, como la sociología, la economía o la pedagogía, y tienen en común el análisis de la variación de la situación de distintas generaciones respecto a diferentes variables socioeconómicas. Las variables más utilizadas han sido la renta, la ocupación o el nivel educativo (Causa y Johansson, 2010; Black y Devereux, 2010). Actualmente la literatura considera tres canales principales de transmisión de capacidades de padres a hijos: la transmisión biológica, la dependencia de la situación económica de los progenitores $\mathrm{y}$, por último, la transmisión directa de la educación (Chevalier, 2004; Chávez, 2015). En este trabajo nos vamos a centrar en la movilidad educativa, considerando la variación que ha experimentado el nivel académico alcanzado por los hijos respecto a sus padres, teniendo en cuenta la situación específica del colectivo inmigrante.

En España se han realizado hasta la fecha numerosos estudios sobre movilidad intergeneracional educativa, que se han diferenciado en función de diversas características de los hijos y de los padres, como la edad o el género (Pascual, 2009; Gil et al., 2010; Moreno, 2011; entre otros). Sin embargo, un elemento aún no considerado es la condición de inmigrante. En este trabajo analizamos la movilidad de este grupo de población en relación con los ciudadanos de origen español. Los resultados obtenidos indican que ser inmigrante afecta negativamente a la probabilidad de alcanzar elevados niveles académicos. Asimismo, se detecta una menor movilidad educativa en el caso de las mujeres inmigrantes.

El trabajo tiene la siguiente estructura: en el segundo apartado, realizamos una breve descripción de los datos que serán utilizados en el resto del documento. En el tercer epígrafe, analizamos cuáles son los determinantes del nivel educativo en España, centrándonos en los que afectan especialmente a la población inmigrante. El cuarto apartado se dedica a estudiar la movilidad educativa intergeneracional, comparando la situación de las personas de origen español con la de los inmigrantes. Finalmente, presentamos las principales conclusiones obtenidas.

\section{Datos}

Para realizar este trabajo hemos utilizado los datos referidos a España de la encuesta EU-SILC (European Union Statistics on Income and Living Conditions) elaborada por Eurostat. En particular, empleamos la muestra transversal de 2011, que contiene un módulo específico sobre transmisión intergeneracional de pobreza. Este módulo ofrece información sobre la familia de cada individuo adulto cuando tenía aproximadamente 14 años de edad, incluyendo variables como el nivel educativo de los padres o su situación laboral, lo que permite estudiar la movilidad intergeneracional desde distintas perspectivas. Además, este módulo aporta información sobre el país de nacimiento y la nacionalidad de los padres, que será fundamental para conocer la situación de la población de origen inmigrante y compararla con el resto de ciudadanos. 
En un artículo reciente de Gil et al. (2010), se ha utilizado la Encuesta de Condiciones de Vida de 2005, que para ese año también cuenta con un módulo de datos intergeneracionales. Aunque en ese trabajo también se analizan los determinantes del nivel educativo y la movilidad intergeneracional, no se ocupa de forma específica del colectivo de personas inmigrantes. También existen estudios que abordan la situación de la población inmigrante a partir de la Encuesta Nacional de Inmigrantes de 2007 (Reher y Silvestre, 2011; Requena y Sánchez-Domínguez, 2011; Aysa-Lastra y Cachón, 2013). Sin embargo, esta encuesta no permite estimar la movilidad educativa, dada la ausencia de información respecto a la educación de los progenitores, ni la comparación entre personas de origen español e inmigrante.

La muestra transversal EU-SILC 2011 cuenta con información acerca de los hogares de los 28 países de la Unión Europea (excepto Irlanda), además de ofrecer datos homogéneos de otros tres países europeos: Suiza, Islandia y Noruega. La muestra completa está basada en 593.997 individuos (34.756 para España). Tomando en consideración que disponemos de información intergeneracional acerca de las personas que tienen una edad comprendida entre los 25 y los 59 años, nuestro trabajo se va a circunscribir a este colectivo, el cual, en términos muestrales, representa a 279.881 individuos a nivel europeo y 16.974 personas residentes en España. Sin embargo, 561 individuos de la muestra española aparecen sin información acerca de su nivel educativo, por lo que finalmente queda reducida a 16.413 individuos.

\section{Determinantes del nivel educativo de los inmigrantes}

\subsection{Metodología}

El estudio de los determinantes del nivel educativo es un tema que cuenta con abundante literatura que ha abordado esta cuestión desde diferentes enfoques. En este trabajo vamos a seguir la aproximación teórica planteada por Lassibille y Navarro (2004), que ha sido utilizada en España en estudios posteriores, como el de Calero et al. (2007). Según esta aproximación, existen cinco grupos de factores que intervienen en el proceso educativo: los recursos del individuo, de sus progenitores, las características de su hogar, las características del establecimiento educativo y el entorno en el aula, y, finalmente, el ambiente social y cultural en que está situado el individuo. $\mathrm{Al}$ igual que en el estudio de Calero et al. (2007), no podemos contar con datos sobre el establecimiento educativo ni sobre el entorno en el aula. Esta limitación, propia de la base de datos empleada, implica una disminución en las posibilidades de identificación de ciertos mecanismos explicativos del rendimiento académico a nivel de centro escolar (existencia de efectos compañeros ${ }^{1} \mathrm{y}$ de gestión, entre otros). Por lo tanto, nuestro trabajo va a tener

1. Los efectos compañeros o peer group efects se refieren a la influencia que tienen las características socioeconómicas de los compañeros de clase sobre los resultados académicos del alumno. 
Tabla 1. Variables utilizadas en el análisis de los determinantes del nivel educativo

\begin{tabular}{lll}
\hline Tipo de variable & Variable teórica & Proxy utilizada \\
\hline Variable dependiente & Logro educativo & Nivel educativo máximo alcanzado por el individuo. Variable categórica \\
& & ordinal (EDUCHIJO) \\
& \\
& 1: educación primaria. \\
& 2: educación secundaria de 1. ${ }^{\text {a }}$ etapa. \\
& 3: educación secundaria de 2. ${ }^{\text {a }}$ etapa. \\
& 4: educación superior.
\end{tabular}

Variables explicativas

\begin{tabular}{|c|c|c|}
\hline \multirow[t]{5}{*}{ Individuo } & Edad & Edad y edad² (EDAD) (EDAD2). \\
\hline & Efecto calendario & Trimestre de nacimiento (TRIM). \\
\hline & Salud & Dummy sobre enfermedad crónica: 1 si padece (ENFCR0). \\
\hline & Género & Dummy sobre género: 0 si es hombre y 1 si es (MUJER). \\
\hline & Inmigrante & $\begin{array}{l}\text { Dummy sobre inmigrante (INMIG): } \\
\text { — } 0 \text { si alguno de los progenitores nació en España. } \\
\text { - } 1 \text { si ambos progenitores o si el único progenitor conocido nacieron } \\
\quad \text { fuera de España. }\end{array}$ \\
\hline \multirow{4}{*}{$\begin{array}{l}\text { Madre y/o padre } \\
\text { (datos a los } 14 \text { años) }\end{array}$} & Variable & Nivel educativo alcanzado por el padre y por la madre. \\
\hline & educativa & $\begin{array}{l}\text { Variable categórica (EDUCMADRE, EDUCPADRE): } \\
0 \text { : sin estudios. } \\
\text { 1: educación primaria y secundaria } 1 \text {. }^{\text {a }} \text { etapa. } \\
\text { 2: educación secundaria } 2 \text {. }^{a} \text { etapa. } \\
\text { 3: educación superior. }\end{array}$ \\
\hline & $\begin{array}{l}\text { Edad de } \\
\text { los padres }\end{array}$ & $\begin{array}{l}\text { Diferencia de edad del padre y de la madre respecto a su hijo o su hija y } \\
\text { diferencia de edad²: }\end{array}$ \\
\hline & & (DIFEDMADRE) (DIFEDMADRE2)(DIFEDPADRE)(DIFEDPADRE2). \\
\hline \multirow[t]{4}{*}{$\begin{array}{l}\text { Hogar } \\
\text { (datos a los } 14 \text { años) }\end{array}$} & $\begin{array}{l}\text { Familia } \\
\text { monoparental }\end{array}$ & Dummy sobre situación monoparental: 1 si (MONOP). \\
\hline & Nivel de renta & $\begin{array}{l}\text { Situación financiera del hogar de origen. Variable categórica (SITUFIN): } \\
\text { 1: muy mala o mala. } \\
\text { 2: moderadamente mala. } \\
\text { 3: moderadamente buena. } \\
\text { 4: buena o muy buena. }\end{array}$ \\
\hline & Trabajo del hogar & Número de personas que trabajaban fuera del hogar (NTRAB). \\
\hline & $\begin{array}{l}\text { Composición } \\
\text { del hogar }\end{array}$ & Número de hermanos en el hogar (HERMANOS). \\
\hline \multirow[t]{2}{*}{ Entorno } & Urbanización & Grado de urbanización. \\
\hline & & $\begin{array}{l}\text { Variable categórica (URBAN): } \\
\text { 1: área densamente poblada. } \\
\text { 2: área intermedia. } \\
\text { 3: área poco poblada. }\end{array}$ \\
\hline
\end{tabular}

Región Comunidad autónoma de residencia (CA). Variable categórica con las 17 CCAA más Ceuta y Melilla.

\footnotetext{
* La variable incluye las siguientes categorías: 1 (educación infantil y educación primaria); 2 (educación secundaria obligatoria); 3 (bachillerato y ciclos formativos de grado medio); 4 (educación universitaria y ciclos formativos de grado superior).

Fuente: elaboración propia en base a EU-SILC (2011).
} 
en cuenta únicamente las características individuales, de los progenitores, del hogar y del entorno.

Los estudios que han tratado los determinantes del nivel educativo del colectivo de inmigrantes también han seguido un esquema muy similar. Entre ellos destaca el trabajo de Bauer y Riphahn (2007), que intenta delimitar cuáles son los factores del nivel educativo para los inmigrantes de segunda generación en Suiza. En dicho trabajo también se tienen en cuenta fundamentalmente las características individuales, del hogar y la región de residencia.

En cuanto a la variable dependiente del modelo, que hace referencia a la educación del individuo, puede presentarse de dos formas distintas. Puede tenerse en cuenta el número de años de escolarización o bien el máximo nivel educativo alcanzado. En nuestro caso, hemos preferido la segunda opción, ya que la muestra EU-SILC brinda información categórica del nivel educativo alcanzado, y abarca desde la educación primaria hasta la educación superior. Al contar con esta variable categórica ordenada, para analizar los determinantes del nivel educativo, lo más adecuado es utilizar estimaciones de máxima verosimilitud con modelos probit o logit ordenados. En nuestro caso, hemos preferido un modelo logit ordenado, ya que nos va a permitir obtener los odds ratios de las distintas variables explicativas.

Las variables que finalmente vamos a considerar aparecen en la tabla 1. Este esquema es similar al expuesto por Calero et al. (2007), aunque también existen diferencias importantes en diversas variables seleccionadas. En primer lugar, la variable dependiente es una variable categórica ordenada del nivel educativo máximo alcanzado. En otros trabajos se ha transformado en una variable continua referida a los años de escolarización. Si bien esta transformación tiene sentido en el caso de personas que han estudiado en un sistema educativo homogéneo, pensamos que en nuestro caso es mejor utilizar una variable discreta, ya que los inmigrantes pueden alcanzar un mismo nivel académico estando escolarizados durante un número distinto de años. Las variables explicativas se han dividido en cuatro bloques que hacen referencia a las características del individuo, las características de sus progenitores, la situación del hogar de origen y el entorno económico y social actual.

Respecto a las características del individuo, se han tenido en cuenta las más habituales, como la edad (también elevada al cuadrado para intentar reflejar una relación no lineal), el trimestre de nacimiento (para tener en cuenta el efecto calendario) el género o el estado de salud. A estas variables hemos añadido una dummy que tiene en cuenta la condición de inmigrante.

A la hora de decidir qué entendíamos por inmigrante, cabían diversas posibilidades. En primer lugar, podíamos basarnos en la nacionalidad o en el país de nacimiento. Aunque ninguna de las dos opciones nos parecía totalmente correcta, hemos preferido utilizar el país de nacimiento, pues pensamos que la nacionalidad está más relacionada con aspectos jurídicos que socioeconómicos. En cualquier caso, todos los estudios que hemos analizado consideran como determinante el país de nacimiento, antes que la nacionalidad. 
En segundo lugar, podíamos entender a los inmigrantes en sentido estricto (aquellos nacidos fuera de España) o bien incluir también a los de segunda generación (individuos nacidos en España cuyos padres nacieron en el extranjero). Finalmente, incluimos también a los inmigrantes de segunda generación, pues pensamos que podían tener un comportamiento educativo distinto a los hijos de españoles. Sin embargo, la inclusión de los inmigrantes de segunda generación introduce problemas de delimitación del conjunto concreto de individuos que se deben considerar como tales. A este respecto, no existe un criterio consensuado sobre la condición de inmigrante, sino que la elección parece depender de las necesidades estadísticas de datos. Card et al. (1998) consideran inmigrante de segunda generación al individuo nacido en el país analizado si tanto el padre como la madre eran inmigrantes, aunque esto restringe considerablemente al grupo de inmigrantes de segunda generación. El mismo criterio utilizan Andersson y Hammarstedt (2011), aunque lo restringen algo más, al exigir que ambos padres hayan nacido en el mismo país, ya que luego realizan una ulterior clasificación en función del país de origen. Por otro lado, Alesina y Guiliano (2010) definen el inmigrante de segunda generación en función del país de origen del padre del entrevistado, con el objetivo de maximizar el número de observaciones. Borjas (1993) utiliza un tercer criterio. Considera inmigrantes de segunda generación a las personas nacidas en el país de referencia si alguno de sus padres ha nacido en un país extranjero. Este criterio es también utilizado por Bauer y Riphahn (2007). En el presente trabajo hemos optado por el primer criterio, es decir, consideramos inmigrantes de segunda generación a los individuos nacidos en España cuyos dos progenitores han nacido fuera de nuestro país. El motivo de esta elección es que los individuos con un progenitor nacido en España y otro en el extranjero son un colectivo muy diverso, con niveles culturales y económicos muy variados, y en todo caso mucho menos homogéneo que las familias con ambos progenitores nacidos en el extranjero. También hemos considerado como inmigrantes a aquellas personas cuyo único progenitor declarado nació fuera de España.

La introducción del país de origen (o de la región geográfica) de los inmigrantes hubiera aportado un valor adicional a este trabajo dada la reconocida heterogeneidad educativa del colectivo inmigrante. Sin embargo, la base de datos empleada únicamente distingue entre tres categorías de países de nacimiento: España, resto de la Unión Europea y resto del mundo. Aunque cabía la posibilidad de haber separado a los inmigrantes en función de su nacimiento dentro o fuera de la Unión Europea, esta diferenciación no ha sido posible debido al escaso número de observaciones que daba lugar a resultados no significativos.

El segundo grupo de variables se refiere a las características de los progenitores. Estas variables han sido obtenidas gracias al módulo de transmisión intergeneracional de pobreza, que proporciona información del hogar de origen cuando el entrevistado contaba aproximadamente con 14 años de edad. La principal variable sería la que se refiere al nivel educativo alcanzado por el padre y por la madre. Esta variable categórica ordinal es similar a la que hemos 
utilizado como variable dependiente, aunque el nivel de detalle es inferior. Para los padres y las madres no podemos distinguir entre los que han cursado solo educación primaria de los que también han estudiado la primera etapa de secundaria. Además de la educación, hemos introducido la edad del padre y la edad de la madre como variables de control. Sin embargo, esta edad está fuertemente correlacionada con la del individuo, por lo que hemos decidido utilizar como variable la diferencia de edad del padre y de la madre respecto a su hijo o su hija.

El tercer bloque de variables pretende considerar la influencia de las características del hogar sobre el nivel educativo del individuo. Utilizando nuevamente el módulo de transmisión intergeneracional de pobreza, se han tenido en cuenta la condición de familia monoparental, la situación financiera del hogar, el número de personas que trabajaban y también el número de hermanos.

Por último, hemos tenido en cuenta el entorno económico y social, considerando el grado de urbanización y la comunidad autónoma de residencia. Sin embargo, conviene realizar una aclaración al respecto. En primer lugar, que estas variables se refieren al momento de la encuesta y no tienen por qué coincidir con la situación de cuando el individuo tenía 14 años. En segundo lugar, es importante destacar que la incorporación de la comunidad autónoma se realiza con el objeto de controlar la estimación de acuerdo con una serie de elementos estructurales de cada uno de sus sistemas educativos y de su estructura económica y social. Por tanto, esta variable se comporta como un indicador sintético de una serie de aspectos múltiples que mejoran la correcta estimación del resto de variables implicadas, pero que no permite realizar un análisis atinado de sus propios resultados.

\subsection{Resultados}

Para comprender las características de las variables utilizadas, exponemos, en la tabla 2, los principales estadísticos descriptivos de todas ellas. Como se ha explicado anteriormente, la muestra finalmente empleada consta de 16.413 observaciones, en las cuales podemos conocer el nivel educativo de los individuos con edades comprendidas entre los 25 y los 59 años. Los estadísticos presentados en la tabla (número de observaciones, media y desviación típica) tienen en cuenta el factor de ponderación establecido en la muestra, por lo que son representativos a nivel poblacional. Asimismo, los resultados totales se han dividido en dos bloques para tener en consideración la característica de inmigrante, fundamental en el presente trabajo. Finalmente, contamos con datos de 14.887 personas consideradas españolas y 1.450 consideradas inmigrantes. Conviene aclarar que hay 76 individuos en la muestra que no podemos categorizar como españoles o inmigrantes, ya que desconocemos el país de nacimiento de su padre y de su madre.

De esta tabla se desprende que cerca del $16 \%$ de la población total solo cuenta con estudios primarios, el 50\% ha completado estudios secundarios (de primer y segundo ciclos) y el $34 \%$ ha finalizado estudios superiores. Si desglo- 
Tabla 2. Estadísticos descriptivos de las variables utilizadas

\begin{tabular}{|c|c|c|c|c|c|c|c|c|c|c|}
\hline \multirow[b]{2}{*}{ Variable } & \multirow[b]{2}{*}{ Valor } & \multicolumn{3}{|c|}{ Total } & \multicolumn{3}{|c|}{ Españoles } & \multicolumn{3}{|c|}{ Inmigrantes } \\
\hline & & $n$ & Media & DT & $n$ & Media & DT & $n$ & Media & DT \\
\hline \multirow[t]{4}{*}{ EDUCHIJO } & 1 & 16.413 & 0,157 & 0,364 & 14.887 & 0,155 & 0,362 & 1.450 & 0,174 & 0,380 \\
\hline & 2 & 16.413 & 0,264 & 0,441 & 14.887 & 0,270 & 0,444 & 1.450 & 0,210 & 0,408 \\
\hline & 3 & 16.413 & 0,239 & 0,426 & 14.887 & 0,228 & 0,419 & 1.450 & 0,337 & 0,473 \\
\hline & 4 & 16.413 & 0,340 & 0,474 & 14.887 & 0,347 & 0,476 & 1.450 & 0,278 & 0,448 \\
\hline EDAD & & 16.413 & 41,25 & 9,51 & 14.887 & 41,59 & 9,55 & 1.450 & 37,95 & 8,46 \\
\hline EDAD2 & & 16.413 & 1.792 & 800 & 14.887 & 1.821 & 805 & 1.450 & 1.511 & 687 \\
\hline TRIMNAC & & 16.412 & 2,488 & 1,109 & 14.887 & 2,487 & 1,108 & 1.449 & 2,493 & 1,111 \\
\hline ENFCRO & & 16.412 & 0,156 & 0,363 & 14.886 & 0,164 & 0,370 & 1.450 & 0,085 & 0,278 \\
\hline MUJER & & 16.413 & 0,497 & 0,500 & 14.887 & 0,494 & 0,500 & 1.450 & 0,525 & 0,500 \\
\hline INMIGRANTE & & 16.337 & 0,095 & 0,293 & 14.887 & 0,000 & 0,000 & 1.450 & 1,000 & 0,000 \\
\hline \multirow[t]{4}{*}{ EDUCMADRE } & 0 & 15.916 & 0,069 & 0,254 & 14.523 & 0,069 & 0,254 & 1.384 & 0,068 & 0,252 \\
\hline & 1 & 15.916 & 0,825 & 0,380 & 14.523 & 0,834 & 0,372 & 1.384 & 0,735 & 0,442 \\
\hline & 2 & 15.916 & 0,057 & 0,232 & 14.523 & 0,052 & 0,222 & 1.384 & 0,103 & 0,304 \\
\hline & 3 & 15.916 & 0,049 & 0,215 & 14.523 & 0,044 & 0,205 & 1.384 & 0,094 & 0,292 \\
\hline \multirow[t]{4}{*}{ EDUCPADRE } & 0 & 15.717 & 0,044 & 0,206 & 14.359 & 0,045 & 0,209 & 1.351 & 0,039 & 0,194 \\
\hline & 1 & 15.717 & 0,790 & 0,407 & 14.359 & 0,798 & 0,400 & 1.351 & 0,715 & 0,452 \\
\hline & 2 & 15.717 & 0,072 & 0,259 & 14.359 & 0,069 & 0,253 & 1.351 & 0,106 & 0,308 \\
\hline & 3 & 15.717 & 0,093 & 0,291 & 14.359 & 0,089 & 0,280 & 1.351 & 0,140 & 0,347 \\
\hline DIFEDMADRE & & 15.080 & 28,47 & 6,25 & 14.623 & 28,68 & 6,004 & 1.232 & 26,40 & 6,88 \\
\hline DIFEDMADRE2 & & 15.080 & 850 & 373 & 14.623 & 859 & 361 & 1.232 & 744 & 394 \\
\hline DIFEDPADRE & & 14.798 & 31,62 & 6,71 & 14.445 & 31,73 & 6,412 & 1.186 & 30,51 & 7,80 \\
\hline DIFEDPADRE2 & & 14.798 & 1.045 & 460 & 14.445 & 1.048 & 439 & 1.186 & 992 & 544 \\
\hline MONOP & & 16.384 & 0,066 & 0,249 & 14.883 & 0,064 & 0,245 & 1.450 & 0,093 & 0,290 \\
\hline \multirow[t]{4}{*}{ SITUFIN } & 1 & 16.223 & 0,123 & 0,329 & 14.749 & 0,117 & 0,322 & 1.433 & 0,178 & 0,382 \\
\hline & 2 & 16.223 & 0,177 & 0,381 & 14.749 & 0,172 & 0,378 & 1.433 & 0,219 & 0,414 \\
\hline & 3 & 16.223 & 0,396 & 0,489 & 14.749 & 0,404 & 0,491 & 1.433 & 0,320 & 0,467 \\
\hline & 4 & 16.223 & 0,304 & 0,460 & 14.749 & 0,306 & 0,461 & 1.433 & 0,283 & 0,451 \\
\hline NTRAB & & 16.049 & 1,773 & 0,833 & 14.602 & 1,767 & 0,834 & 1.412 & 1,829 & 0,815 \\
\hline HERMANOS & & 16.107 & 2,336 & 1,390 & 14.651 & 2,294 & 1,344 & 1.416 & 2,758 & 1,723 \\
\hline \multirow[t]{3}{*}{ URBAN } & 1 & 16.413 & 0,523 & 0,499 & 14.887 & 0,514 & 0,500 & 1.450 & 0,609 & 0,488 \\
\hline & 2 & 16.413 & 0,221 & 0,415 & 14.887 & 0,221 & 0,415 & 1.450 & 0,215 & 0,411 \\
\hline & 3 & 16.413 & 0,256 & 0,436 & 14.887 & 0,265 & 0,442 & 1.450 & 0,176 & 0,381 \\
\hline \multirow[t]{19}{*}{$\overline{C C A A}$} & AND & 16.413 & 0,179 & 0,383 & 14.887 & 0,188 & 0,391 & 1.450 & 0,100 & 0,300 \\
\hline & ARA & 16.413 & 0,028 & 0,165 & 14.887 & 0,027 & 0,161 & 1.450 & 0,039 & 0,195 \\
\hline & AST & 16.413 & 0,023 & 0,151 & 14.887 & 0,025 & 0,157 & 1.450 & 0,008 & 0,092 \\
\hline & BAL & 16.413 & 0,024 & 0,152 & 14.887 & 0,021 & 0,143 & 1.450 & 0,050 & 0,218 \\
\hline & CAN & 16.413 & 0,047 & 0,211 & 14.887 & 0,047 & 0,212 & 1.450 & 0,040 & 0,196 \\
\hline & CNT & 16.413 & 0,013 & 0,112 & 14.887 & 0,013 & 0,113 & 1.450 & 0,012 & 0,107 \\
\hline & CAT & 16.413 & 0,152 & 0,359 & 14.887 & 0,145 & 0,352 & 1.450 & 0,224 & 0,417 \\
\hline & CLM & 16.413 & 0,045 & 0,207 & 14.887 & 0,046 & 0,210 & 1.450 & 0,032 & 0,177 \\
\hline & CYL & 16.413 & 0,053 & 0,224 & 14.887 & 0,056 & 0,230 & 1.450 & 0,026 & 0,160 \\
\hline & CEU & 16.413 & 0,002 & 0,042 & 14.887 & 0,002 & 0,041 & 1.450 & 0,003 & 0,050 \\
\hline & EXT & 16.413 & 0,022 & 0,148 & 14.887 & 0,024 & 0,154 & 1.450 & 0,005 & 0,069 \\
\hline & GAL & 16.413 & 0,058 & 0,234 & 14.887 & 0,062 & 0,241 & 1.450 & 0,015 & 0,120 \\
\hline & LR & 16.413 & 0,007 & 0,083 & 14.887 & 0,006 & 0,080 & 1.450 & 0,011 & 0,105 \\
\hline & MAD & 16.413 & 0,141 & 0,348 & 14.887 & 0,128 & 0,334 & 1.450 & 0,273 & 0,445 \\
\hline & MEL & 16.413 & 0,001 & 0,035 & 14.887 & 0,001 & 0,031 & 1.450 & 0,003 & 0,055 \\
\hline & MUR & 16.413 & 0,033 & 0,178 & 14.887 & 0,034 & 0,180 & 1.450 & 0,023 & 0,149 \\
\hline & NAV & 16.413 & 0,014 & 0,117 & 14.887 & 0,014 & 0,117 & 1.450 & 0,014 & 0,118 \\
\hline & PV & 16.413 & 0,047 & 0,212 & 14.887 & 0,049 & 0,215 & 1.450 & 0,031 & 0,173 \\
\hline & VAL & 16.413 & 0,111 & 0,314 & 14.887 & 0,113 & 0,316 & 1.450 & 0,091 & 0,288 \\
\hline
\end{tabular}

Fuente: elaboración propia en base a EU-SILC (2011). 
samos estos datos entre españoles e inmigrantes, podemos comprobar que estos últimos cuentan, como media, con más estudios secundarios de segunda etapa (un 33,7\%), pero con menos estudios superiores (un 27,8\%). Este resultado parece indicar una preferencia de los inmigrantes por estudios que permitan una más rápida integración en el mercado laboral. Sin embargo, la base de datos empleada no permite conocer las motivaciones de las personas a la hora de elegir estudios, por lo que solamente podemos avanzar esta interpretación como una hipótesis. También puede observarse cómo el porcentaje de personas que han alcanzado únicamente estudios primarios también es ligeramente superior entre los inmigrantes.

En el primer bloque de variables explicativas, referidas a características personales, podemos encontrar ciertas diferencias en función del origen del individuo. En primer lugar, la media general de edad está en torno a los 41 años, aunque los inmigrantes son algo más jóvenes (38 años). También hay ligeras diferencias en cuanto al género, ya que las mujeres inmigrantes superan el $52 \%$, mientras que en el resto de la población están situadas ligeramente por debajo del 50\%. Donde sí encontramos datos radicalmente distintos es en el estado de salud, ya que el $16,4 \%$ de los españoles padece algún tipo de enfermedad crónica, mientras que en el colectivo de inmigrantes este porcentaje se reduce prácticamente a la mitad (un $8,5 \%$ ). Por último, la variable inmigrante nos indica qué porcentaje de la población consideramos inmigrante de acuerdo con nuestra calificación. Este porcentaje se sitúa en el 9,5\%. De este colectivo de inmigrantes, un $25 \%$ procede de países de la Unión Europea y el restante $75 \%$, de otros países.

Respecto a las características de los padres, observamos en primer lugar que el nivel educativo de ambos progenitores, tanto si tomamos a los individuos españoles como a los inmigrantes, es muy inferior al de sus hijos, aunque también conviene aclarar que el nivel educativo alcanzado por las madres es claramente inferior al de los padres. Resulta especialmente llamativo que las madres y los padres de los inmigrantes hayan alcanzado un nivel educativo más elevado que los progenitores de los individuos españoles. Esta situación se debe a que España es un país en el que el acceso generalizado a la educación secundaria y, sobre todo, a la educación superior es un fenómeno relativamente reciente. Al considerar solamente a los padres y a las madres de los individuos que tienen entre 25 y 59 años, estamos ante personas cuya media de edad se sitúa en torno a los 70 años y que tuvieron escasas oportunidades en el sistema educativo español. Por último, la diferencia de edad de los hijos con sus madres, como cabría esperar, es ligeramente inferior a la diferencia con los padres.

Las características del hogar de origen también son distintas para individuos nacionales e inmigrantes. Conviene destacar que, a los 14 años, los inmigrantes vivían en mayor proporción en hogares monoparentales (un 9,3\%), tenían una situación financiera más delicada (el 17,8\% la consideraba mala o muy mala), contaban con un número mayor de trabajadores por hogar (un 1,83) y tenían más hermanos (un 2,76 incluido el individuo). Por último, las dos variables del entorno muestran que la población inmigrante se concentra en zonas urbanas 
(un 60,9\%) y también que está más representada en determinadas comunidades autónomas, como Madrid y Cataluña.

Una vez expuestos los estadísticos descriptivos, a continuación llevamos a cabo un análisis multivariante de los determinantes del nivel educativo a través de un modelo logit ordenado. Como paso previo a este análisis, hemos querido corregir un problema que se nos planteaba con algunas variables que presentaban valores perdidos (missing values). En concreto, los mismos se han estimado mediante el método de imputación por regresión recomendado por la OECD (2008) para los casos de la edad del padre y de la madre, del número de trabajadores del hogar y del número de hermanos, con porcentajes reducidos de información perdida. Sin embargo, no hemos querido estimar los valores missing en aquellas variables que consideramos fundamentales en nuestro modelo, como las que hacen referencia al nivel de estudios de los progenitores o la condición de inmigrante. Tras esta imputación, nuestra muestra queda reducida a un total de 15.318 observaciones, lo que nos supone perder 1.095 observaciones, el $6,7 \%$ del total. Asimismo, se ha comprobado que las variables imputadas mantengan la distribución de sus observaciones en relación con la situación original.

En la tabla 3 se presentan los resultados de todas las variables explicativas del modelo, incluyendo tanto los coeficientes como los odds ratios, aunque estos últimos solo se presentan para las variables que resultan significativas. Los odds ratios nos indican cómo se altera la relación entre la probabilidad de una situación y la probabilidad de la situación de referencia cuando aumenta en una unidad el valor de la variable considerada, manteniendo constantes las demás. Así, valores de un odd ratio superiores a 1 suponen crecimientos de la relación de probabilidades, mientras que valores inferiores a 1 suponen decrecimientos.

En las dos primeras columnas aparecen los resultados de las 15.318 observaciones, mientras que en las siguientes columnas se presentan los resultados desagregados. Para ello se ha dividido la muestra entre españoles e inmigrantes de acuerdo con nuestra clasificación y se ha vuelto a realizar la estimación para estos dos grupos siguiendo el mismo modelo logit ordenado. Esta desagregación nos permitirá conocer si el nivel educativo de los inmigrantes depende de las mismas variables que el nivel educativo del resto de individuos.

En primer lugar, la mayoría de las variables que reflejan la situación del individuo son significativas para el conjunto de la población y mantienen el signo previsto. La edad influye de forma positiva en el logro educativo, lo que puede ser debido a que algunos individuos siguen estudiando a partir de los 25 años. Las personas que padecen una enfermedad crónica tienen menos probabilidades de alcanzar un nivel de estudios más elevado y las mujeres logran un mayor nivel educativo que los hombres. El trimestre de nacimiento es la única variable cuya influencia no es significativa. En cuanto a los inmigrantes, su logro educativo es menor que el obtenido por los ciudadanos de origen español, siendo su odd ratio 0,699 , lo que significa que ser inmigrante reduce las probabilidades académicas en un 30,1\%. Sin embargo, si desagregamos la muestra y estimamos el modelo únicamente para los inmigrantes, ninguna de las variables individuales resulta significativa. 
Tabla 3. Logit ordenado del nivel educativo alcanzado

\begin{tabular}{|c|c|c|c|c|c|c|}
\hline \multirow[b]{2}{*}{ Variable explicativa } & \multicolumn{2}{|c|}{ Residentes $(n=15.318)$} & \multicolumn{2}{|c|}{ Nacionales $(n=14.028)$} & \multicolumn{2}{|c|}{ Inmigrantes $(n=1.290)$} \\
\hline & Coefic. & Odds ratios & Coefic. & Odds ratios & Coefic. & Odds ratios \\
\hline$\overline{E D A D}$ & $0,137^{* t}$ & 1,147 & $0,132^{* *}$ & 1,142 & 0,064 & \\
\hline EDAD2 & $-0,002^{* *}$ & 0,998 & $-0,002^{\star \star}$ & 0,998 & $-0,001$ & \\
\hline TRIMNAC & 0,012 & & 0,015 & & $-0,041$ & \\
\hline ENFCRO & $-0,297^{* *}$ & 0,743 & $-0,303^{* *}$ & 0,738 & $-0,172$ & \\
\hline MUJER & $0,200^{* *}$ & 1,221 & $0,218^{* *}$ & 1,243 & 0,029 & \\
\hline INMIGRANTE & $-0,363^{* *}$ & 0,695 & \multicolumn{4}{|c|}{ Variable omitida } \\
\hline EDUCMADRE $=0$ & \multicolumn{6}{|c|}{ Categoría de referencia } \\
\hline EDUCMADRE $=1$ & $1,099^{* *}$ & 3,002 & $1,076^{\star \star}$ & 2,932 & $1,397^{\star *}$ & 4,034 \\
\hline EDUCMADRE = 2 & $1,739^{* *}$ & 5,693 & $1,729^{\star \star}$ & 5,635 & $1,998^{* *}$ & 7,372 \\
\hline EDUCMADRE $=3$ & $2,293^{* *}$ & 9,908 & $2,130^{\star *}$ & 8,412 & $3,172^{* *}$ & 23,849 \\
\hline EDUCPADRE $=0$ & \multicolumn{6}{|c|}{ Categoría de referencia } \\
\hline EDUCPADRE = 1 & $0,330^{* *}$ & 1,390 & $0,391^{\star \star}$ & 1,478 & $-0,334$ & \\
\hline EDUCPADRE = 2 & $1,164^{* *}$ & 3,204 & $1,276^{\star \star}$ & 3,581 & 0,368 & \\
\hline EDUCPADRE $=3$ & $1,832^{* *}$ & 6,247 & $1,898^{* *}$ & 6,674 & $1,190^{*}$ & 3,288 \\
\hline DIFEDPADRE & 0,024 & & 0,029 & & $-0,011$ & \\
\hline DIFEDPADRE2 & 0,000 & & 0,000 & & 0,000 & \\
\hline MONOP & $-0,017$ & & $-0,086$ & & 0,457 & \\
\hline$\overline{\text { SITUFIN }=1}$ & \multicolumn{6}{|c|}{ Categoría de referencia } \\
\hline SITUFIN = 2 & $0,379^{* *}$ & 1,462 & $0,318^{* \star}$ & 1,375 & $0,869^{* *}$ & 2,384 \\
\hline SITUFIN = 3 & $0,789^{* *}$ & 2,202 & $0,769^{* *}$ & 2,158 & $0,764^{* *}$ & 2,147 \\
\hline SITUFIN = 4 & $1,006^{* *}$ & 3,735 & $0,972^{* *}$ & 2,644 & $1,163^{* *}$ & 3,200 \\
\hline NTRAB & $-0,159^{* *}$ & 0,853 & $-0,156^{* *}$ & 0,856 & $-0,155$ & \\
\hline HERMANOS & $-0,117^{* *}$ & 0,889 & $-0,117^{\star \star}$ & 0,890 & $-0,114^{* \star}$ & 0,892 \\
\hline URBAN $=1$ & \multicolumn{6}{|c|}{ Categoría de referencia } \\
\hline URBAN $=2$ & $-0,290^{* *}$ & 0,748 & $-0,305^{\star *}$ & 0,737 & $-0,312$ & \\
\hline URBAN = 3 & $-0,422^{* *}$ & 0,655 & $-0,449^{\star \star}$ & 0,638 & $-0,120$ & \\
\hline Madrid & \multicolumn{6}{|c|}{ Categoría de referencia } \\
\hline Andalucía & $-0,403^{* *}$ & 0,669 & $-0,444^{* *}$ & 0,642 & 0,140 & \\
\hline Aragón & $-0,103$ & & $-0,095$ & & $-0,220$ & \\
\hline Asturias & $-0,211^{\star}$ & 0,810 & $-0,231^{\star}$ & 0,793 & $-0,263$ & \\
\hline Baleares & $-0,545^{* *}$ & 0,580 & $-0,575^{\star *}$ & 0,563 & $-0,443$ & \\
\hline Canarias & $-0,469^{* *}$ & 0,626 & $-0,487^{\star *}$ & 0,615 & $-0,575$ & \\
\hline Cantabria & $-0,264^{*}$ & 0,768 & $-0,277^{\star}$ & 0,758 & $-0,217$ & \\
\hline C. La Mancha & $-0,287^{\star *}$ & 0,750 & $-0,282^{\star *}$ & 0,755 & $-0,403$ & \\
\hline C. y León & $-0,180$ & & $-0,188$ & & $-0,399$ & \\
\hline Cataluña & $-0,298^{* *}$ & 0,743 & $-0,350^{\star \star}$ & 0,704 & $-0,094$ & \\
\hline Extremadura & $-0,313^{* *}$ & 0,731 & $-0,342^{\star *}$ & 0,711 & 1,140 & \\
\hline Ceuta & $-0,946^{* *}$ & 0,388 & $-1,001^{\star \star}$ & 0,368 & $-0,806$ & \\
\hline Galicia & $-0,080$ & & $-0,110$ & & 0,433 & \\
\hline La Rioja & $-0,398^{* *}$ & 0,672 & $-0,360^{* \star}$ & 0,697 & $-0,583^{*}$ & 0,558 \\
\hline Melilla & $-0,626$ & & 0,419 & & $-1,475^{*}$ & 0,229 \\
\hline Murcia & $-0,817^{* *}$ & 0,442 & $-0,846^{\star \star}$ & 0,429 & $-0,544$ & \\
\hline Navarra & 0,016 & & 0,018 & & $-0,107$ & \\
\hline P. Vasco & $0,207^{*}$ & 1,230 & $0,262^{*}$ & 1,299 & $-0,894$ & \\
\hline C. Valenciana & $-0,546^{* *}$ & 0,579 & $-0,568^{\star \star}$ & 0,567 & $-0,372$ & \\
\hline
\end{tabular}

* Significativa al $95 \%$.

** Significativa al $99 \%$.

Fuente: elaboración propia en base a EU-SILC (2011). 
En segundo lugar, las variables que tienen en cuenta las características de los progenitores demuestran tener una fuerte influencia sobre el logro educativo de los hijos. La variable más importante es la que se refiere a la educación de la madre, puesto que influye de forma positiva en todos los residentes en España, pero resulta especialmente trascendental en el colectivo de inmigrantes. Como puede verse en la última columna, tener una madre con estudios superiores aumenta la probabilidad de alcanzar un nivel educativo superior casi 25 veces. El nivel educativo del padre también ejerce un efecto positivo, aunque los odds ratios obtenidos indican que su influencia no es tan importante. Además, esta variable solo resulta significativa para el colectivo de inmigrantes cuando el padre tiene estudios universitarios. La diferencia de edad respecto a los progenitores solo es significativa en el caso de la madre, con un efecto positivo pero de pequeña cuantía.

Las variables que se refieren a las características del hogar ejercen el efecto esperado sobre el nivel educativo alcanzado, tanto para los hijos de españoles como para los inmigrantes, con la excepción de la variable que tiene en cuenta la condición de familia monoparental, que no resulta significativa. En cuanto a la situación financiera del hogar de origen, cuanto mejor es, mayor es el logro educativo de los hijos. Por otro lado, las familias más numerosas pueden dedicar menos recursos a la educación de cada uno de sus hijos, por lo que desciende el nivel educativo alcanzado a medida que aumenta la cantidad de hermanos. Finalmente, el número de personas que trabajan en el hogar ejerce también un efecto negativo sobre el nivel educativo, lo que puede deberse a una menor dedicación por parte de los progenitores que trabajan fuera del hogar respecto al control y seguimiento de la formación de sus hijos. Sin embargo, esta variable no resulta significativa en el colectivo de inmigrantes.

Por último, las variables del entorno muestran una influencia importante en los individuos de nacionalidad española. Cuanto mayor sea el grado de urbanización, mayor será la probabilidad de completar estudios superiores. Además, la residencia en algunas comunidades autónomas tendrá una influencia significativa sobre la posibilidad de continuar estudiando. Sin embargo, estas variables dejan de ser significativas si nos centramos únicamente en el colectivo de inmigrantes. Pensamos que esto es debido a que el grado de urbanización y la comunidad autónoma se refieren al momento de la encuesta y no al momento en el que el individuo recibió la educación. En un país como España, donde existe escasa movilidad espacial, se puede esperar que las personas nacidas en nuestro país sigan viviendo en el mismo entorno y en la misma región donde estudiaron. Por lo tanto, estas dos variables siguen siendo significativas. Por el contrario, para los inmigrantes el grado de urbanización de la población donde crecieron no tiene por qué guardar relación con el de su residencia actual (Pérez et al., 2014). Esta situación aún es más evidente con la variable comunidad autónoma, ya que los inmigrantes de primera generación nacieron fuera de España. Conviene reiterar las dificultades existentes para interpretar los resultados obtenidos respecto a la comunidad de residencia, dado su comportamiento como indicador sintético multifactorial. 
En resumen, el nivel educativo alcanzado por la población de origen inmigrante es más reducido que el de los ciudadanos de origen español y depende de variables distintas. Aunque hay diversas variables significativas, como la edad de la madre, el número de hermanos o el número de personas que trabajan fuera del hogar, las dos que tienen mayor influencia son el nivel educativo de la madre y la situación financiera del hogar de origen, por lo que es de esperar que el nivel de movilidad intergeneracional sea más reducido en este colectivo.

\section{Movilidad intergeneracional educativa de los inmigrantes}

\subsection{Metodología}

La movilidad intergeneracional educativa ha sido tratada por numerosos autores siguiendo técnicas muy distintas. Calero et al. (2007) y Mediavilla y Calero (2010) realizan una tipificación basándose en tres grandes categorías: la correlación bivariante, la regresión y las matrices de transición. En el presente trabajo se estimaran las tres aproximaciones dada su complementariedad.

En primer lugar, se encuentran las técnicas de correlación que intentan determinar la relación directa existente entre el nivel educativo de padres e hijos. Estas técnicas, aparentemente sencillas, tienen varias referencias en la literatura. Podemos citar a Lillard y Willis (1994) para Malasia; Österberg (2000) para Suecia, Binder y Woodruff (2002) para México, Hertz et al. (2008) para 42 países alrededor del mundo, Mediavilla y Calero (2010) para varios países latinoamericanos y Ranasinghe (2015) para Australia. En cuanto a España, encontramos los siguientes trabajos: Carabaña (1999), Sánchez (2004), Calero y Escardíbul (2005), Gil et al. (2010) y Moreno (2011).

Un segundo grupo de técnicas es la regresión, que puede realizarse a partir de diferentes estimaciones: modelos lineales, mínimos cuadrados ordinarios bietápicos, variables instrumentales o estimaciones de máxima verosimilitud. Si bien la literatura es muy amplia, aquí solo citamos algunos trabajos como los de Comi (2003); Sánchez (2004); Calero et al. (2007); Pascual (2009); Gil et al. (2010) y Mediavilla y Calero (2010). La variable de logro educativo más usual ha sido la que se refiere a los años de escolaridad (en el caso de variable continua) o al nivel educativo máximo alcanzado (en el caso de variable discreta). En cuanto a las variables independientes, se considera la educación de los padres y las madres, así como algunas variables de control lineales y cuadráticas (Behrman y Taubman, 1990; Solon, 1992).

La tercera alternativa metodológica es la utilización de matrices de transición. La misma consiste en calcular una matriz cuadrada en la que cada elemento representa la probabilidad de que un individuo alcance un determinado nivel educativo dado el nivel educativo alcanzado por su padre o por su madre. $\mathrm{Al}$ tratarse de una matriz cuadrada, la diagonal representa los casos donde existe inmovilidad, mientras que el resto de elementos de la matriz indicaría que se ha producido movilidad (ascendente o descendente). Para construir la matriz, se estima un modelo de máxima verosimilitud (logit ordenado), que tiene 
como variable dependiente la que se refiere al nivel educativo alcanzado por los hijos y como principal variable explicativa, el nivel educativo de los padres. Usualmente, se introducen variables de control para tener en cuenta la edad de los hijos y la de los padres (mediante de la diferencia de edad entre padres e hijos). Algunas de las aplicaciones empíricas que han empleado esta técnica son los trabajos de Corak y Heisz (1999) y Fortin y Lefebvre (1998) para Canadá; Björklund y Jäntti (1997) y Österberg (2000) para Suecia; Peters (1992) para los Estados Unidos; Dearden et al. (1997) para Gran Bretaña; Mediavilla y Calero (2010) para Latinoamérica y, por último, los estudios antes citados de Sánchez (2004) y Gil et al. (2010) para España.

Aunque la movilidad educativa puede realizarse respecto a ambos progenitores, nosotros solamente vamos a tener en cuenta a la madre. Para tomar esta decisión, nos hemos basado en los resultados obtenidos en el apartado anterior, que demostraban una mayor influencia del nivel educativo de la madre sobre el de los hijos. En cuanto a los hijos, hemos vuelto a dividir a los individuos de la muestra entre inmigrantes y españoles para poder tener estimaciones diferenciadas. Además, para conocer si existen diferencias de movilidad entre géneros, hemos calculado las matrices de transición de forma separada, distinguiendo entre hijos e hijas.

Por último, con el fin de tener matrices cuadradas y calcular índices de movilidad, hemos reclasificado el nivel educativo de los hijos de acuerdo con las cuatro categorías que contemplamos para las madres:

— Sin estudios (SINEST).

- Educación primaria y secundaria $10^{a}$ etapa (PRIM).

- Educación secundaria de 2. ${ }^{\mathrm{a}}$ etapa y postsecundaria no superior (SECUN).

- Educación superior (SUPER).

Esta nueva clasificación conlleva un problema metodológico, ya que en la primera categoría (sin estudios) aparecen observaciones referentes al nivel educativo de la madre, pero ninguna de las personas encuestadas (hijos e hijas) ha manifestado estar situada en la primera categoría. Una posible solución a este problema hubiese sido unir esta categoría con la siguiente, pero pensamos que de esta forma estaríamos infravalorando la movilidad académica que realmente se ha producido. Hemos preferido mantener estas cuatro categorías asumiendo que en todos los casos en que la madre no tenga estudios se va a producir movilidad educativa ascendente.

\subsection{Resultados}

En primer lugar, se presentan los resultados obtenidos a partir del análisis de correlación entre el nivel educativo alcanzado por la madre y el obtenido por sus hijos, distinguiendo en función del género de estos (véase la tabla 4).

En el caso de los hijos varones, existe un resultado similar entre aquellos considerados nacionales respecto de los inmigrantes, si bien estos últimos muestran una correlación ligeramente mayor. En cambio, en el caso de las 
Tabla 4. Análisis de correlación

\begin{tabular}{lcccccc}
\hline & \multicolumn{2}{c}{ Nivel educativo de los hijos } & & \multicolumn{2}{c}{ Nivel educativo de las hijas } \\
\cline { 2 - 3 } \cline { 5 - 6 } & Españoles & Inmigrantes & & Españolas & Inmigrantes \\
\hline Nivel educativo de la madre & 0,3228 & 0,3876 & & 0,3352 & 0,4537 \\
\hline
\end{tabular}

Fuente: elaboración propia en base a EU-SILC (2011).

hijas, la correlación es mucho más elevada en las inmigrantes, lo que indica una menor movilidad intergeneracional educativa.

En segundo lugar, hemos realizado un análisis de regresión a través de una aproximación multivariante (logit ordenado). La variable dependiente sigue siendo la que se refiere al nivel educativo alcanzado por el hijo o por la hija y como independiente consideramos el nivel educativo de la madre, así como las variables de control que tienen en cuenta la edad del hijo y la diferencia de edad respecto a la madre (véase la tabla 5).

A nivel general, los resultados indican que la educación de la madre influye en la educación de sus hijos e hijas y que el grado de influencia se incrementa en la medida en que crece su educación (como puede comprobarse en la tabla, todos los odds ratios son superiores a la unidad y van creciendo con el nivel educativo de la madre). Además, existen diferencias en cuanto al género de los hijos y en cuanto a su condición de inmigrante. Las personas que se ven más influidas por el nivel educativo materno son las hijas de origen español, especialmente cuando se consideran estudios primarios y secundarios. Por el contrario, en las mujeres inmigrantes, la educación materna no tiene tanta influencia, salvo en el caso de que se trate de estudios universitarios. En el caso

Tabla 5. Análisis de regresión de la movilidad educativa

\begin{tabular}{|c|c|c|c|c|}
\hline \multirow[b]{2}{*}{ Variables } & \multicolumn{2}{|c|}{ Nivel educativo de los hijos } & \multicolumn{2}{|c|}{ Nivel educativo de las hijas } \\
\hline & Españoles & Inmigrantes & Españolas & Inmigrantes \\
\hline SINEST MADRE & \multicolumn{4}{|c|}{ Categoría de referencia } \\
\hline PRIM MADRE & $4,97^{* *}$ & $5,59^{* *}$ & $6,88^{* *}$ & $3,00^{* *}$ \\
\hline SECUN MADRE & $18,66^{* *}$ & $21,06^{* *}$ & $27,66^{* *}$ & $9,87^{* *}$ \\
\hline SUPER MADRE & $33,05^{* *}$ & $33,67^{* *}$ & $69,57^{* *}$ & $75,06^{* *}$ \\
\hline EDAD & $1,06^{*}$ & 1,03 & $1,10^{\text {** }}$ & 1,00 \\
\hline EDAD2 & $1,00^{* *}$ & 1,00 & $1,00^{*+*}$ & 1,00 \\
\hline DIFEDADMADRE & $1,20^{* *}$ & 1,14 & $1,17^{* *}$ & $1,20^{*}$ \\
\hline DIFEDADMADRE2 & $1,00^{* *}$ & 1,00 & $1,00^{* *}$ & $1,00^{* *}$ \\
\hline$N$ & 7.078 & 633 & 7.408 & 740 \\
\hline Prob $>\mathrm{Chi}^{2}$ & 0,000 & 0,000 & 0,000 & 0,000 \\
\hline
\end{tabular}

Los coeficientes están expresados en odds ratios.

* Significativa al $95 \%$.

** Significativa al 99\%.

Fuente: elaboración propia en base a EU-SILC (2011). 
de los varones, no existen grandes diferencias entre hijos de origen nacional o inmigrante.

En tercer lugar, las matrices de transición obtenidas para madres e hijos y para madres e hijas se reproducen en la tabla 6 , donde se distingue entre la población de origen español de aquella que hemos calificado como inmigrante. En la segunda parte de la tabla se han calculado los principales indicadores de movilidad para los cuatro grupos considerados. Para obtener estos valores, hemos realizado estimaciones de máxima verosimilitud a través de modelos logit ordenados.

Las matrices indican, a nivel agregado, que los hijos y las hijas superan los niveles educativos alcanzados por sus madres, lo que implica una movilidad educativa ascendente fruto de la incorporación masiva de la población al sistema educativo. Como ejemplo, cabe citar que, para la categoría sin estudios, existen casos de madres, pero no de hijos o hijas. En el caso de que la madre alcance el nivel educativo de secundaria, existen altos porcentajes de movilidad ascendente y aquí se detecta una primera diferencia entre nacionales e inmigrantes. Claramente, los primeros muestran una movilidad mayor. Por último, una madre con educación superior implica una mayor probabilidad de que sus hijos lleguen a este nivel académico. En este caso, dicho condicionante es más fuerte en el caso de las hijas.

Tabla 6. Matrices de transición e indicadores de movilidad

\begin{tabular}{|c|c|c|c|c|c|c|c|c|}
\hline \multicolumn{9}{|c|}{ Españoles } \\
\hline \multirow{2}{*}{$\begin{array}{c}\text { Nivel educativo } \\
\text { MADRE }\end{array}$} & \multicolumn{4}{|c|}{ Nivel educativo del hijo } & \multicolumn{4}{|c|}{ Nivel educativo de la hija } \\
\hline & SINEST & PRIM & SECUN & SUPER & SINEST & PRIM & SECUN & SUPER \\
\hline SINEST & 0 & 80,57 & 11,61 & 7,81 & 0 & 81,65 & 11,24 & 7,11 \\
\hline PRIM & 0 & 45,51 & 24,87 & 29,62 & 0 & 39,27 & 26,24 & 34,49 \\
\hline SECUN & 0 & 18,19 & 20,55 & 61,26 & 0 & 13,86 & 18,23 & 67,91 \\
\hline SUPER & 0 & 11,15 & 15,16 & 73,69 & 0 & 6,01 & 9,80 & 84,19 \\
\hline \multicolumn{9}{|c|}{ Inmigrantes } \\
\hline \multirow{2}{*}{$\begin{array}{c}\text { Nivel educativo } \\
\text { MADRE }\end{array}$} & \multicolumn{4}{|c|}{ Nivel educativo del hijo } & \multicolumn{4}{|c|}{ Nivel educativo de la hija } \\
\hline & SINEST & PRIM & SECUN & SUPER & SINEST & PRIM & SECUN & SUPER \\
\hline SINEST & 0 & 79,79 & 15,69 & 4,53 & 0 & 69,86 & 22,55 & 7,59 \\
\hline PRIM & 0 & 41,37 & 37,67 & 20,96 & 0 & 43,62 & 36,62 & 19,75 \\
\hline SECUN & 0 & 15,79 & 34,26 & 49,95 & 0 & 19,02 & 36,20 & 44,78 \\
\hline \multirow[t]{3}{*}{ SUPER } & 0 & 10,50 & 28,03 & 61,48 & 0 & 3,00 & 10,96 & 86,05 \\
\hline & & & & & \multicolumn{2}{|c|}{ Españoles } & \multicolumn{2}{|c|}{ Inmigrantes } \\
\hline & & & & & Hijos & Hijas & Hijos & Hijas \\
\hline \multicolumn{5}{|c|}{ Índice de inmovilidad } & 0,3494 & 0,3542 & 0,3428 & 0,4147 \\
\hline \multicolumn{5}{|c|}{ Movilidad ascendente } & 215,75 & 228,64 & 208,58 & 201,16 \\
\hline \multicolumn{5}{|c|}{ Movilidad descendente } & 44,49 & 29,67 & 54,31 & 32,97 \\
\hline
\end{tabular}

Fuente: elaboración propia en base a EU-SILC (2011). 
El cálculo del índice de inmovilidad nos muestra, para el caso de la población española, que la inmovilidad educativa es muy similar independientemente del género de los hijos, lo que representa alrededor del $35 \%$ de la población. Sin embargo, en el caso de las hijas, se produce mayor movilidad ascendente y menor descendente, lo que supone una clara mejora de nivel educativo de las hijas respecto de sus madres. En el colectivo de inmigrantes, los valores por géneros son diferentes, puesto que existe una menor movilidad en el caso de las hijas. Este resultado va en la línea del obtenido mediante el análisis de correlación.

Si comparamos la situación de los españoles con la de los inmigrantes, los resultados son muy diferentes en función del género. Los varones de ambos colectivos presentan indicadores de movilidad muy similares, mientras que las mujeres se comportan de forma distinta. Como puede comprobarse, las mujeres de origen inmigrante presentan una movilidad educativa muy inferior a las de origen español.

\section{Conclusiones}

Los trabajos que analizan los determinantes del logro educativo y la movilidad intergeneracional son abundantes en la literatura, con diversos estudios empíricos que analizan el caso español. El presente trabajo intenta profundizar en las circunstancias especiales en que se encuentra la población inmigrante y conocer si existen diferencias importantes respecto al resto de ciudadanos que residen en nuestro país. La base de datos utilizada ha sido la muestra transversal EU-SILC de 2011, que contiene un módulo de transmisión intergeneracional de pobreza.

El análisis de los determinantes del nivel educativo en nuestro país, realizado mediante una estimación de máxima verosimilitud, demuestra que la variable acerca de la condición de inmigrante es una de las que afectan al logro académico de los ciudadanos y de las que disminuyen la probabilidad de alcanzar niveles elevados. Evidentemente, la condición de inmigrante actuaría como un indicador sintético de una serie de condicionantes (sociales, económicos y culturales) que afectarían a la educación de los individuos.

Tras constatar esta situación, hemos estimado el mismo modelo únicamente para la población de origen inmigrante, a fin de conocer si los determinantes del nivel educativo son diferentes a los del resto de individuos. En el caso de la población inmigrante, encontramos dos variables que van a tener un efecto crucial sobre el nivel educativo. La primera variable es la que muestra el nivel educativo alcanzado por la madre, y la segunda, la situación financiera del hogar de origen. En este punto es importante recordar que la base de datos no contaba con información del centro docente.

Una vez detectada la influencia del nivel educativo de la madre, hemos calculado la movilidad intergeneracional académica de los inmigrantes para poder compararla con los ciudadanos nacidos en España. Para ello hemos realizado un análisis de correlación, una estimación vía regresión y el cálculo 
de matrices de transición educativa de madres a hijos y de madres a hijas para ambos colectivos.

El análisis de correlación demuestra una menor movilidad educativa en el caso de las mujeres inmigrantes. Asimismo, la estimación vía regresión indica una clara influencia de la educación de la madre en las posibilidades educativas de sus hijos y/o hijas, con un impacto superior en el caso de las mujeres nacionales. Finalmente, los indicadores de movilidad nos muestran unos resultados muy similares en el caso de los varones, independientemente de su país de origen. Sin embargo, la movilidad es mucho más reducida en el caso de mujeres inmigrantes.

Esta inmovilidad observada indicaría que las mujeres inmigrantes se encuentran afectadas por algunos condicionantes adicionales al de sus pares hombres. Existiría una cuestión de género que se sumaría a la condición de inmigrante. Sin conocer las situaciones particulares, podemos afirmar que dicho colectivo debería recibir un tratamiento específico por parte de las políticas públicas por dos motivos principales: en primer lugar, para detectar el origen de esta desventaja relativa y, en segundo lugar, para aplicar las políticas necesarias a fin de asegurar una igualdad real de oportunidades educativas.

Finalmente, los resultados obtenidos para el caso español también pueden compararse con los resultados de otros países europeos gracias a la base de datos utilizada que contiene datos homogéneos para toda la Unión Europea, lo cual constituye una futura línea de investigación.

\section{Referencias bibliográficas}

Alesina, A. y Giuliano, P. (2010). «The power of the family». Journal of Economic Growth, 15, 93-125. <https://doi.org /10.1007/s10887-010-9052-z>

ANDERSSON, L. y HammarstedT, M. (2011). «Transmission of self-employment across immigrant generations: The importance of ethnic background and gender». Review of Economics of the Household, 9, 555-577. $<$ https://doi.org/10.1007/s11150-010-9102-5>

ARANGO, J. (2004). «La inmigración en España a comienzos del siglo XXI». En: LEAL MALDONADO, J. (coord.). Informe sobre la situación demográfica en España. Madrid: Fundación Fernando Abril Martorell.

AYSA-LASTRA, M. y CACHÓN, L. (2013). «Determinantes de la movilidad ocupacional segmentada de los inmigrantes no comunitarios en España». Revista Internacional de Sociología, 71, 383-413. <https://doi.org/10.3989/ris.2012.05.03>

Bauer, P. y RipHAHN, R.T. (2007). «Heterogeneity in the intergenerational transmission of educational attainment: Evidence from Switzerland on natives and second-generation immigrants». Journal of Population Economics, 20, 121-148. <https://doi.org/10.1007/s00148-005-0056-5>

Behrman, J. y TaUbman, P. (1990). «The Intergenerational Correlation between Children's Adult Earnings and their Parent's Income: Results from the Michigan Panel Survey of Income Dynamics». Review of Income and Wealth, 36, 115-127. $<$ https://doi.org/10.1111/j.1475-4991.1990.tb00275.x> 
BeINE, M. et al. (2014). «Measuring Immigration Policies: Preliminary Evidence from IMPALA». CESifo Working Paper 5109. Center for Economic Studies \& Ifo Institute, Munich. <https://doi.org/10.1093/cesifo/ifu038>

Binder, M. y WoOdrufF, C. (2002). «Inequality and Intergenerational Mobility in Schooling: The case of Mexico». Economic Development and Cultural Change, 20, 249-267. <https://doi.org/10.1086/322882>

BJÖRKLUnd, A. y JÄNTTI, M. (1997). «Intergenerational Income Mobility in Sweden Compared to the United States». American Economic Review, 87, 1009-1018. <http://www.jstor.org/stable/2951338>.

Black, S. y DeVereux, P. (2010). «Recent Developments in Intergenerational Mobility». IZA Discussion Paper Series, 4866. IZA. Bonn. <https://doi.org/10.3386/w15889>

BORJAS, G. (1993). "The Intergenerational Mobility of Immigrants». Journal of Labor Economics, 11, 113-135. <https://doi.org/10.1086/298319>

CAlero, J. et al. (2007). Desigualdades socioeconómicas en el sistema educativo español. Madrid: Ministerio de Educación y Ciencia.

Calero, J. y Escardíbul, J.O. (2005). «Financiación y desigualdades en el sistema educativo y de formación profesional de España». En: NAVArro, V. (dir.). La situación social de España. Madrid: Biblioteca Nueva-Fundación Largo Caballero.

CARABAÑA, J. (1999). Dos estudios sobre movilidad intergeneracional. Madrid: Fundación Argentaria-Visor.

CARD, D.; Dinardo, J. y Estes, E. (1998). «The more things change: Immigrants and the children of immigrants in the 1940s, the 1970s, and the 1990s». NBER Working Paper, 6519. Cambridge, MA: National Bureau of Economic Research. <https://doi.org/10.3386/w6519>

Carrasco, R.; Jimeno, J.F. y Ortega, A.C. (2008). "The Effect of Immigration on the Labor Market Performance of Native-born Workers: Some Evidence for Spain». Journal of Population Economics, 21 (3), 627-648. <https://doi.org/10.1007/s00148-006-0112-9>

Causa, O. y Johansson, Å. (2010). «Intergenerational Social Mobility in OECD Countries». OECD Journal: Economic Studies, 6 (1), 33-76. <https://doi.org/10.1787/eco_studies-2010-5km33scz5rjj>

CHÁveZ, F. (2015). «Intergenerational transmission of education: The relative importance of transmission channels». Latin American Economic Review, 24 (1), 1-44. <https://doi.org/10.1007/s40503-014-0015-1>

Chevalier, A. (2004). "Parental education and child's education: A natural experiment». IZA Discussion Paper Series, 1153. IZA. Bonn.

ComI, S. (2003). «Intergenerational Mobility in Europe: Evidence from ECHP». Working Papers del Dipartimento de Economia Politica e Aziendale, 3. Milán. Università degli Studi di Milano.

CORAK, M. y HeISZ, A. (1999). «The Intergenerational Earnings and Income Mobility of Canadian Men: Evidence from Longitudinal Income Tax Data. The Journal of Human Resources, 34, 504-533. <https://doi.org/10.2139/ssrn.139768>

DeARden, L.; MACHIN, S. y ReEd, H. (1997). «Intergenerational Mobility in Britain». The Economic Journal, 107, 47-66. <http://www.jstor.org/stable/2235270>. 
Eurostat (2011). Migrants in Europe: A statistical portrait of the first and second generation. Luxemburgo: European Union.

- (2012). European Union Statistics on Income and Living Conditions. EU-SILC 2011 longitudinal (rev.4 / August 2016).

- (2015). Migration and migrant population statistics. Luxemburgo: European Union. Recuperado el 4 de marzo de 2015, de <http://ec.europa.eu/eurostat/statisticsexplained/index.php/Migration_and_migrant_population_statistics $>$.

ForTin, N. y LEFEBVRE, S. (1998). «Intergenerational Income Mobility in Canada». En: CORAK, M. (dir.). Labour Markets, Social Institutions, and the Future of Canada's Children's. Ottawa: Statistics Canada.

GARrido, L. (2005). «La inmigración en España». En: GonZÁlez RodríGuez, J.J. y ReQuena, M. (eds.). Tres décadas de cambio social en España. Madrid: Alianza.

Gil, M.; PABlos, L. de y Martínez, M. (2010). «Los determinantes socioeconómicos de la demanda de educación superior en España y la movilidad educativa intergeneracional». Hacienda Pública Española: Revista de Economía Pública, 193, 75-108.

GonZÁleZ, L. y OrTega, F. (2011). «How Do Very Open Economies Absorb Large Immigration Flows?: Recent Evidence from Spanish Regions». Labour Economics, 18 (1), 57-70. <https://doi.org/10.1016/j.labeco.2010.06.001>

Hertz, T. et al. (2008). «The Inheritance of Educational Inequality: International Comparisons and Fifty Year Trends». Journal of Economic Analysis \& Policy, 7 (2), $1935-1682$.

<https://doi.org/10.2202/1935-1682.1775>

Lassibille, G. y Navarro, L. (2004). Manual de economía de la educación: Teoría y casos prácticos. Madrid: Pirámide.

LILlaRD, L. y WiLLIS, R. (1994). «Intergenerational Educational Mobility: Effects of Family and State in Malaysia». The Journal of Human Resources, 29, 1126-1166. <https://doi.org/10.2307/146136>

Mediavilla, M. y Calero, J. (2010). «Movilidad educativa en Latinoamérica: Un estudio para seis países». Revista Española de Educación Comparada, 16, 287-303. <https://doi.org/10.5944/reec.16.2010.7533>

Moreno, A. (2011). «La reproducción intergeneracional de las desigualdades educativas: Límites y oportunidades de la democracia». Revista de Educación, n. ${ }^{\circ}$ extraordinario, 183-206.

OECD (2008). Handbook on constructing composite indicators: Methodology and user guide. París: OECD.

Österberg, T. (2000). «Intergenerational Income Mobility in Sweden: What do Tax-Data Show?». Review of Income and Wealth, 46, 421-36. <https://doi.org/10.1111/j.1475-4991.2000.tb00409.x>

PASCUAL, M. (2009). "Intergenerational income mobility: The transmission of socio-economic status in Spain». Journal of Policy Modeling, 31, 835-846. <https://doi.org/10.1016/j.jpolmod.2009.07.004>

PÉreZ, M.; JiméneZ, M. y Blanco, M. (2014). "Análisis de las diferencias regionales en España aplicado al fenómeno de la inmigración durante la crisis». Boletín Económico del ICE, 3051, 39-51.

PETERS, E. (1992). «Patterns of Intergenerational Mobility in Income and Earnings». The Review of Economics and Statistics, 74, 456-66. <https://doi.org/10.2307/2109490> 
Ranasinghe, R. (2015). "The Transmission of Education across Generations: Evidence from Australia». Journal of Economic Analysis \& Policy, 15 (4), 1893-1917. <https://doi.org/10.1515/bejeap-2014-0139>

REHER, D. y SilveSTRE, J. (2011). "Internal migration patterns of foreign-born immigrants in Spain: A study based on the National Immigrant Survey (ENI-2007)». Revista Internacional de Sociología. Monográfico n. ${ }^{\circ}$ 1, 167-188. <https://doi.org/10.3989/ris.2011.iM1.390>

REQUENA, M. y REHER, D. (2009). «La creciente experiencia inmigratoria en España». En: RequenA, M. y ReHer, D. (eds.). Las múltiples caras de la inmigración en España. Madrid: Alianza.

Requena, M. y SÁnCHeZ-Domínguez, M. (2011). "Las familias inmigrantes en España». Revista Internacional de Sociología, 69, 79-104. <https://doi.org/10.3989/ris.2011.iM1.387>

Rica, S. de la; GlitZ, A. y Ortega, F. (2014). «Immigration in Europe: Trends, Policies and Empirical Evidence». En: CHISwICK, B. y Miller, P. (eds.). Handbook of the Economics of International Migration. Oxford: North Holland.

SÁNCHEZ, A. (2004). "Movilidad intergeneracional de ingresos y educativa en España (1980-90)». Document de treball 2004/1. Barcelona: Institut d'Economia de Barcelona.

Solon, G. (1992). «Intergenerational Income Mobility in the United States». American Economic Review, 82, 393-408. <http://www.jstor.org/stable/2117312>. 
